\title{
Tip of the Red Giant Branch Distances. I. Optimization of a Maximum Likelihood Algorithm
}

\author{
Dmitry Makarov ${ }^{1,2,3}$, Lidia Makarova ${ }^{1,2}$, Luca Rizzi and R. Brent Tully \\ Institute for Astronomy, University of Hawaii, 2680 Woodlawn Drive, Honolulu, HI 96822 \\ and \\ Andrew E. Dolphin \\ Steward Observatory, University of Arizona, Tucson, AZ 85721 \\ and \\ Shoko Sakai \\ Division of Astronomy and Astrophysics, University of California, Los Angeles, \\ CA 90095-1562 \\ and \\ Edward J. Shaya \\ University of Maryland, Astronomy Department, College Park, MD 20743 \\ Received —
}

\footnotetext{
${ }^{1}$ also Special Astrophysical Observatory of the Russian Academy of Sciences, Nizhnij Arkhyz, 369167, Karachaevo-Cherkessia, Russia

${ }^{2}$ Isaac Newton Institute of Chile, SAO Branch

${ }^{3}$ Observatoire de Lyon, 9, avenue Charles André, 69561, St-Genis Laval Cedex, France
} 


\begin{abstract}
Accurate distances to galaxies can be determined from the luminosities of stars at the Tip of the Red Giant Branch (TRGB). We use a Maximum Likelihood algorithm to locate the TRGB in galaxy color-magnitude diagrams. The algorithm is optimized by introducing reliable photometric errors and a completeness characterization determined with artificial star experiments. The program is extensively tested using Monte-Carlo simulations, artificial galaxies, and a sample of nearby dwarf galaxies observed with HST/WFPC2 and ACS. Our procedure is shown to be reliable, to have good accuracy, and to not introduce any systematic errors. The methodology is especially useful in cases where the TRGB approaches the photometric limit and/or the RGB is poorly populated.
\end{abstract}

Subject headings: galaxies: distances and redshifts - methods: data analysis 


\section{Introduction}

In the last decade, significant progress has been made in the study of the large-scale structure of the Universe. Projects such as the Sloan Digital Sky Survey are dramatically improving our knowledge of the kinematics, dynamics and structure in the distribution of galaxies at large distances. At the same time, tremendous advances have been made on the small scale of the local neighborhood as a result of searches for nearby galaxies through wide-field optical (Karachentseva \& Karachentsev 1998, 2000; Whiting et al. 2002), and blind HI surveys (Meyer et al. 2004). In the decade since the publication of a list of 226 galaxies with radial velocities less than $500 \mathrm{~km} \mathrm{~s}^{-1}$ (Karachentsev 1994), the number of

known nearby galaxies has doubled (Karachentsev et al. 2004). The Hubble Space Telescope (HST) and new large ground-based telescopes make it possible to study stellar populations and measure accurate distances to these nearby galaxies.

The luminosity of the tip of red giant branch (TRGB) provides a standard candle that is being used to give accurate distances to galaxies within $10 \mathrm{Mpc}$ (Karachentsev et al. 2005; Maíz-Apellániz et al. 2002). Over the last five years, members of our team have participated in two snapshot surveys of nearby galaxies using WFPC2 aboard HST, which have provided us with the material for distances of $\sim 10 \%$ accuracy for about 150 nearby galaxies. Further significant progress has been made with HST/ACS pointed observations.

The TRGB method is comparable in accuracy with the Cepheid Period-Luminosity distance indicator (Sakai et al. 1996; Bellazzini et al. 2001). Furthermore, since essentially all resolved galaxies are found to contain old stars, the TRGB method is applicable to galaxies of all morphological types, including spiral, elliptical and irregular galaxies. The TRGB method also demands less telescope time to acquire a good distance (only one HST/WFPC2 orbit is required for a TRGB detection in galaxies situated within 5 Mpc; HST/ACS gives a TRGB detection for galaxies out to $10 \mathrm{Mpc}$ in one orbit). From early 
applications involving simply the visual identification of the termination of the red giant branch, there has been a succession of more sophisticated techniques to improve the reliability and accuracy of the TRGB measurement (Lee et al. 1993; Sakai et al. 1996; Mendez et al. 2002).

About 200 galaxies have already been observed with HST/WFPC2 and HST/ACS, either by our collaboration or by others, with data suitable for a TRGB determination in the HST archive. This outstanding material includes images of galaxies of essentially all types, though dwarfs predominate. The galaxies are mostly within $6 \mathrm{Mpc}$. There are systems with high and low metallicities and mixes of stellar types. Some targets have the TRGB situated well above the photometric limit but many have the TRGB only about 1 mag above the photometric limit. The main aim of this work is to improve the TRGB estimation accuracy closer to the photometric limit. The existing sample gives us an excellent opportunity to compare TRGB detection results for galaxies of different types and observed in different ways. Ultimately, it is our intention to apply a consistent methodology to the analysis of all the relevant data in the HST archive.

\section{Methodology}

Baade (1944) found that direct photographs of galaxies in the Local Group showed the presence of a background sheet of red stars and suggested that the brightest stars in this sheet are like the brightest globular cluster giant branch stars. Sandage (1971) pointed out that the brightest red stars in the underlying sheets of M 31, M 33, and IC 1613 have the same absolute magnitude, $M_{V} \approx-3.0 \pm 0.2 \mathrm{mag}$.

According to modern stellar evolution theory, the tip of the first-ascent red giant branch marks the violent onset of core-helium burning in low-mass stars. Observationally, 
this phenomenon causes a distinct and abrupt termination of the bright end of the red giant branch luminosity function. This discontinuity translates directly into an excellent distance indicator: the bolometric luminosity of the TRGB for low-mass stars is predicted to vary by only about 0.1 mag for ages ranging from 2 up to 15 Gyr (Iben \& Renzini 1983) and for metallicities encompassing the entire range represented by Galactic globular clusters of $-2.1<[\mathrm{Fe} / \mathrm{H}]<-0.7$ (Salaris \& Cassisi 1997). The discontinuity is also found empirically to be stable at the $\sim 0.1 \mathrm{mag}$ level in the $I$ band for the same set of stellar properties. A detailed review of the TRGB method is given by Madore \& Freedman (1998) and Sakai (1999). Here we briefly describe the main features of the method and recent improvements.

Early applications of the TRGB method relied on a visual estimate of the TRGB luminosity from a color-magnitude diagram (CMD). Lee et al. (1993) defined the position of the TRGB in a reproducible and quantitative manner. They used a standard imageprocessing edge-detection (ED) algorithm employing the zero-sum Sobel kernel $[-2,0,+2]$ which, when convolved with the stellar luminosity function, gives a maximum in its output at the luminosity where the count discontinuity is the greatest. The measuring errors for the tip magnitude $I_{T R G B}$ were estimated to be typically $0.1-0.2 \mathrm{mag}$ in their study. The method as calibrated at that time is applicable over the range of metallicities -2.2 dex $<[\mathrm{Fe} / \mathrm{H}]<-0.7$ dex.

A disadvantage of the binned approach of Lee et al. is that the TRGB solution depends both on the luminosity function (LF) bin size and bin placement. Sakai et al. (1996) modified this method for application with a smoothed, continuous LF. The continuous $I$-band LF is generated by replacing the discretely distributed stellar magnitudes with the Gaussian-smoothed function:

$$
\Phi(m)=\sum_{i=1}^{N} \frac{1}{\sqrt{2 \pi} \sigma_{i}} \exp \left[-\frac{\left(m_{i}-m\right)^{2}}{2 \sigma_{i}^{2}}\right]
$$

where $m_{i}$ and $\sigma_{i}$ are the magnitude and photometric error of the $i$ th star and $N$ is the total 
number of stars in the sample. The ED filter is then defined by

$$
E(m)=\Phi\left(I+\bar{\sigma}_{m}\right)-\Phi\left(I-\bar{\sigma}_{m}\right)
$$

where $\bar{\sigma}_{m}$ is the mean photometric error for all stars with magnitudes between $m-0.05$ and $m+0.05$.

The ED method can be very effective for galaxies when the TRGB is located more than 2 mag above the magnitude limit of the photometry, because in such cases the photometric errors at the tip are modest and crowding and incompleteness are not significant. The current estimate of uncertainties in TRGB measurements if well above the photometric limit is 0.05 mag (Sakai et al. 2004). However, the method becomes less precise if sampling statistics are poor or incompleteness strongly affects the LF within 1 mag of the TRGB location. In such cases, the edge-detector response becomes noisy even when the TRGB is clearly seen in the CMD.

As an alternative, a maximum-likelihood (ML) TRGB detection method was proposed by Mendez et al. (2002) (hereafter M02). The ED algorithms find a maximum of the first derivative of the luminosity function of the stars in the TRGB region, whereas in the ML analysis a predefined luminosity function is fitted to the observed distribution of the stars. The ED method has the advantage that it is model independent. However the ED algorithm works by differentiation of the function determined by an ensemble of points which need to be smoothed. Smoothing with a small window gives a noisy result whereas smoothing with a large window can introduce a bias. The ML method does not have this disadvantage, but we need to know the stellar luminosity distribution function and photometric errors distribution for an adequate probability estimation. The ML method proposed by M02 and a similar method by Sakai et al. (2004) do not account for systematic errors introduced by crowding effects, Malmquist bias, and incompleteness.

Here we introduce the further development of the ML method for the determination of 
the TRGB where we account for systematic photometric errors. Artificial star experiments provide a reliable and well established method of photometric error estimation. The HSTphot package (Dolphin 2000) which we used for the photometry contains a powerful and flexible procedure for conducting artificial star tests. Experiments give us a measure of photometric errors from the differences between input and output magnitudes of artificial stars, and of the completeness function from the input recovery rate. The completeness, mean photometric error, and dispersion in error are determined as a function of magnitude and affect the maximum likelihood luminosity function fit.

\section{Maximum Likelihood (ML) TRGB detection}

The maximum likelihood method in our work is mathematically similar to the method introduced by M02. There are two main differences. The first, already mentioned, is the use of a photometric errors function defined from artificial star experiments. It has been mentioned by many authors (see, for example, Gallart et al. 1996 and Dolphin 2002) that artificial star tests are the only accurate way to solve the problem of photometric errors, blending, and incompleteness. The procedure of artificial star tests involves the generation of a very large library of artificial stars that cover the necessary range of magnitudes and colors on the CMD so that the distribution of recovered photometry is adequately sampled. The photometry program analyzes the generated artificial stars with the same routines and parameter selections as for observed stars. Photometric error estimations are determined by comparison of predefined input magnitudes with photometered magnitudes.

The second main difference from M02 is the parameterization of the RGB luminosity function. For the "theoretical" luminosity function, we assume a simple power-law with a cut-off in the TRGB region plus a power-law of a second slope for a stellar population 
brighter than the TRGB.

$$
\psi= \begin{cases}10^{a\left(m-m_{T R G B}\right)+b}, & m-m_{T R G B} \geq 0 \\ 10^{c\left(m-m_{T R G B}\right)}, & m-m_{T R G B}<0\end{cases}
$$

M02 use the slope $a=0.3$, which is also used in some other studies, but we allow some flexibility to the choice of the parameter $a$. From our experience there are moderate variations around a slope $\sim 0.3$. Filtering with color constraints can affect the slope. The tests performed in this study do not confine the slope parameter $a$ but in the summary there will be a re-evaluation of this issue.

Definition of the photometric errors lead to the LF smoothing:

$$
\varphi(m)=\int \psi\left(m^{\prime}\right) \rho\left(m^{\prime}\right) e\left(m \mid m^{\prime}\right) d m^{\prime}
$$

where $\rho(m)$ is the completeness function and $e\left(m \mid m^{\prime}\right)$ is the error distribution function. For the latter, we assume a Gaussian distribution and take into account the bias in the photometric error function; i.e. we use the first two moments of the distribution:

$$
e\left(m \mid m^{\prime}\right)=\frac{1}{\sqrt{2 \pi} \sigma\left(m^{\prime}\right)} \exp \left(-\frac{\left(m-\bar{m}\left(m^{\prime}\right)\right)^{2}}{2 \sigma^{2}\left(m^{\prime}\right)}\right)
$$

Then the probability of a star detection of magnitude $m_{i}$ within interval $d m$ is:

$$
P_{i}=\frac{\varphi\left(m_{i} \mid \mathbf{x}\right) d m}{\int_{m_{\min }}^{m_{\max }} \varphi(m \mid \mathbf{x}) d m}
$$

where $m_{\min }$ and $m_{\max }$ define the region of the validity of the parameterized distribution function and $\mathbf{x}$ is a vector of parameters. Therefore, the probability of a given realization is $P=\prod_{i=1}^{N} P_{i}$. Let us define the minimization function as $\mathcal{L}=-\ln P$ and drop therms independent of model parameters, then:

$$
\mathcal{L}=-\sum_{i=1}^{N} \ln \varphi\left(m_{i} \mid \mathbf{x}\right)+N \ln \int_{m_{\min }}^{m_{\max }} \varphi(m \mid \mathbf{x}) d m
$$

Thus, in this method there are four parameters for the minimization, $\mathbf{x}\left(m_{T R G B}, a, b, c\right)$. The completeness function $\rho$ and photometric error function $e$ are defined from the artificial star tests. 


\subsection{A Clean TRGB measurement}

The input data to our program includes observed and artificial star photometry results. The observed photometry is extracted from HST/WFPC2 images with HSTphot (Dolphin 2000) or from HST/ACS images with the modification DOLPHOT (Dolphin 2006). From the artificial star tests we calculate statistics on mean deviations, dispersions, and completeness from the distribution of differences between recovered and input $V$ and $I$ star magnitudes. We use Gaussian weighting instead of binning for a smoothed behavior of our functions. Our experience show that an 0.1-0.2 mag window size preserves the original completeness behavior.

As an example of our procedure, consider the color-magnitude diagram of DDO 70 shown in Fig.1. Colors and magnitudes are in the HST flight filters F814W, which transforms well to Cousins $I$, and F606W, which transforms adequately to Johnson $V$. The most prominent feature of the CMD is the RGB reaching more than 3 mag brighter than the photometric cutoff. Stars immediately above the RGB are part of the asymptotic giant branch (AGB). There is a modestly populated main sequence at F606W-F814W $\sim 0$. The peak of the first derivative of the Gaussian smoothed luminosity function of stars provides a first guess of the location of the TRGB. The dashed box in the CMD isolates the stars which were used in the LF construction. The magnitude limits are set \pm 1 mag brighter and fainter than the preliminary estimated value of the TRGB. The left color limit creates the restriction $\mathrm{F} 606 W-\mathrm{F} 814 W>0.4$. These magnitude and color limits are adjusted to suit the particular CMD. The blue color limit is selected to eliminate main sequence stars.

There is no red color limit for a more subtle reason. In instances where the TRGB lies within a magnitude or so of the photometric limit, the dashed box will encroach on this limit. In fact there are two photometric limits to consider because there are two filter bands. There is the F814W magnitude limit which is a horizontal cut on the CMD and 
there is the F606W magnitude limit which causes a slanting cut on the CMD. If we insist that only stars with color information can contribute to the LF then the slanting color cut creates a bias in the population of the LF. The bias can be avoided if the LF is allowed to include stars clipped from the CMD because they are very red; ie, stars detected in the F814W filter but too faint to have been detected in the F606W filter. Note that the point is moot in the case of DDO 70 because the analysis is conducted at magnitudes well above the photometric limit. This example demonstrates that the lack of a red limit has little effect on the definition of the LF because the number of stars redward of the RGB is small compared with the number of stars in the RGB.

The current discussion does not give consideration to reddening, either foreground or internal to the target galaxies. This important topic will be considered in Paper II of this series.

The output parameters - TRGB value, RGB jump, RGB slope and AGB slope - and their uncertainties, are calculated from the analysis leading to the ML function fit. Error intervals are $68 \%$ confidence level. Results in the case of DDO 70 are given in the caption to Fig. 1. A detailed discussion of the method uncertainties is given in the next section.

\section{Tests}

\subsection{Recovering the TRGB with Monte-Carlo tests}

We performed extensive tests of internal precision through Monte-Carlo simulations with known model parameters. The model luminosity function was defined by random stars distributed according to Eq. 3. Tests were run with this function defined by 37,371 , 3706 and 37064 stars within 1 mag of the TRGB. The photometric limit for our WFPC2 data is $I \sim 25.5 \mathrm{mag}$ and this limit defines the range of our tests. In each case there were 
subtests with distance modulus varied such that $I_{T R G B}$ was shifted from 22.0 mag to 25.5 mag in steps of 0.25 mag. Each subtest contained 1000 realizations. The parameters $a, b$ and $c$ were the same for all the tests: $a=0.3, b=0.3$ and $c=0.2$; quite similar to real galaxy LF parameters. We used the completeness function and photometric errors model from real galaxy photometry (DDO 226: discussed later) to mimic real observations. Such tests allowed us to study the influence of the number of observed stars and the problems that arise as the TRGB approaches the photometric limit.

An example of a recovered LF is given in Fig.2 where the input TRGB is one magnitude above the photometric limit. Median deviations of the recovered TRGB values from the input values are given in Fig.3. The four panels of the figure report results according to the different number of the stars introduced into the LF. The figure shows that our method does not introduce any bias even when the TRGB is close to the photometric limit and it gives good accuracy even in cases of small star samples. An exception is only the extremely poor populated diagram where the TRGB is close to the photometric limit. In Fig.4 we show the distribution of reconstructed $I_{T R G B}$ for 6 different sets of tests. It can be noted that the recovered $I_{T R G B}$ has a roughly Gaussian distribution except in cases with very few stars or very close to the photometric limit. The other three parameters of the model luminosity function are also recovered well, except in the limiting cases of few stars or near the photometric limit. As an example, Fig.5 shows the recovery of the RGB LF slope parameter.

Fig.6 shows the distribution of the measured TRGB uncertainty vs. photometric errors of the individual stars for different numbers of input stars. It can be seen from the figure that ML method accuracy is strongly dependent on the number of stars. The error of TRGB reconstruction becomes comparable to the photometric error of individual stars if there are about 1000 stars in the analysis. We can not expect an accuracy better than 0.1 
mag if there are less than 300 stars in the first magnitude below the TRGB.

\subsection{Comparison with other procedures using artificial galaxies}

To test our TRGB detection algorithm further, we intercompare some of the most commonly used methods and the ML procedure presented here on an additional set of artificial galaxy constructions that resemble real galaxies. We have applied the TRGB methods to synthetic color-magnitude diagrams (CMD's) constructed using the ZVAR code (Bertelli et al. 1992). This code is based on the Padova stellar evolution tracks (Girardi et al. 2000), and performs interpolation both in age and metallicity to produce a smooth distribution of stars.

As an aside, note that single-orbit ACS data extends $\sim 1.5$ mag deeper in $I$ band than snapshot WFPC2 data, corresponding to equal sensitivity at a factor $\sim 2$ greater distance. Since we have both WFPC2 and ACS material at our disposition, we explore tests tailored separately for the two cameras and with our most common exposures times. The differences in sensitivity of the two cameras are to be appreciated in comparing real and simulated WFPC2 and ACS data. A direct comparison of WFPC2 and ACS data in a common field is given in Section 4.5. The tests to be discussed in this section are based on the photometric errors and the completeness effects recovered using artificial star experiments based on real single-orbit observations obtained with HST/ACS.

To avoid biases, no attempt at modeling the errors or the completeness function was made, and the artificial stars were drawn from a look-up table (see Rizzi et al. 2002, for details). Three different star formation histories were adopted, the first consisting of an old single episode about 14 Gyr ago (closely reproducing the case of a globular cluster), the second based on continuous star formation activity from 14 Gyr ago until now, and the 
third producing a mostly young galaxy. The model galaxies were put at distance moduli ranging from 28 to 30, the latter being the extreme case for a TRGB detection in a single orbit with HST/ACS. The TRGB detection methods we have tested are the ED (applied to both the linear and the logarithmic LF), and the ML (both in the version of Mendez et al. 2002, and in the version presented in this paper). The results of our simulations are shown in Figure 7. The figure shows the contribution to the total distance error due to the TRGB detection alone. A significant difference in the behavior of the methods we tested is apparent from these tests. For the nearest cases all the methods give very good results. For more distant galaxies, ED methods seem to be more affected by problems connected with degraded photometric quality and by increased bias (stars preferentially scatter toward brighter magnitudes because the LF increases toward fainter magnitudes).

The right and bottom panels of Figures 7 show what happens for a galaxy with a very strong AGB contamination, obtained by using a continuous star formation history, or a star formation that increases over time. In these cases, the RGB is superimposed on an extended and bright AGB component.

Comparison of the upper left panel of Figure 7 with the other two panels clearly

shows that all methods are basically insensitive to the presence of AGB stars, and that ML methods produce significantly smaller errors at all distances and for all the combinations of star formation histories.

\subsection{Poorly populated CM diagrams}

To consider the effect of poorly populated CM diagrams in greater detail, we investigated the case of a globular cluster-like galaxy, at an intermediate distance of $(m-M)_{0}=28$. The star population in the CM diagram is quantified by the number of 
stars in the first magnitude bin after the tip (hereafter, $N^{(-1)}$ ). We generated galaxies with $N^{(-1)}$ between 300 and 30 and applied the detection methods to all cases. Results are shown in Figure 8.

The left panel of Figure 8 shows that there is a general trend for a large scatter in the TRGB detection when $N^{(-1)}$ falls below 100. This result is consistent with previous studies (Madore \& Freedman 1995). Careful scrutiny of this plot also shows that the average dispersion of measurements is significantly lower at any $N^{(-1)}$ for ML methods, compared to ED methods. This point is further demonstrated by the right panel of Figure 8, which shows the r.m.s. of TRGB detections against $N^{(-1)}$. Not only is the r.m.s. of ED methods always higher, but ED methods tend to break down at a $N^{(-1)}$ of about 100 stars, while ML methods seem to be able to produce reasonable results at least down to 50 stars. Incidentally, we note that we do not find in our simulations the large systematic deviations pointed out by Madore \& Freedman (1995). The difference is most likely due to the presence in our simulations of AGB stars. Madore \& Freedman (1995) use fiducial lines from globular clusters populated with power-law distributions, so they have no stars brighter than the TRGB. Removing stars from their simulated galaxies can only produce fainter TRGB detections, while our use of synthetic stellar populations produced with stellar models results in deviations in both directions.

In conclusion, we verify that ML appears to perform better than ED methods especially when the level of the TRGB approaches the detection limit. We also verify that even a prominent AGB component does not significantly affect the detection. Finally, we applied the methods to poorly populated diagrams, and found that ED methods produce significantly higher errors for each level of $N^{(-1)}$. ML methods seem to be able to detect the TRGB with as few stars as $N^{(-1)}=50$. 


\subsection{Comparison of ML and ED procedures using real data}

To estimate the external uncertainties of our method we compared the results of ML TRGB detection with the results of the modified ED algorithm (Sakai et al. 1996) for the same objects. For the comparison we selected 10 dwarf galaxies from the snapshot surveys (HST programs 8601 and 8192). We have taken both dIrr and dSph dwarfs at different distances, extending to $5 \mathrm{Mpc}$. The stellar photometry was done with the software HSTphot (Dolphin 2000) in the course of the snapshot programs with artificial star photometry added. The WLM galaxy (DDO 221) was included in the sample to provide a very nearby case with a deep CMD. The HST/WFPC2 observation of DDO 221 were made within HST programs 6898 and 6813, and photometry results along with the results of artificial star experiments were taken from J. Holtzman's HST Local Group stellar photometry archive (Holtzman et al. 2005, http://astronomy.nmsu.edu/holtz/archival/html/lg.html).

The results of the comparison of the two methods are presented in Fig.9. The figure shows $I_{T R G B}$ measured with the ML method vs. $I_{T R G B}$ measured with ED method. The horizontal error bars are the ML one-sigma errors and the vertical bars are the equivalent errors with the ED method.

The agreement of the two measurements for two fields in DDO 221 are good. The mean difference between two methods is 0.07 mag, whereas the difference between the two

fields measured with the same method is 0.05 for the ML and 0.19 for the ED method. For many of the galaxies the TRGB is quite close to the photometric limit which challenges both methods. A marked disagreement between the two methods can be seen in the case of KK 35 where it is likely that the ED measurement is picking up the onset of the AGB. The maximum likelihood estimates are expected to be more effective in cases where the TRGB lies close to the photometric limit. It should be noted that the photometric error bars are smaller for the ML method in most cases. The maximum likelihood procedure 
has an advantage due to the incorporation of reliable photometric errors and completeness function through the artificial star experiments. We did not find any correlation of $I_{T R G B}$ (maximum likelihood) and $I_{T R G B}(\mathrm{ED})$ difference with the morphological type or absolute magnitude of the sample galaxies.

In general, the comparison of the maximum likelihood and ED methods shows that both methods work well, with comparable accuracy, when the RGB is well-populated and is situated at least 2 mag above the photometric limit. However, when the TRGB is closer to the photometric limit and/or poorly populated, the ED measurement can give a result with poor accuracy and bias. On the other hand, tests suggest that the ML method, combined with a proper description of incompleteness and real photometric errors, gives reliable results whenever the TRGB is accessible.

\subsection{A comparison between two fields in the same galaxy}

It is interesting to compare the ML TRGB detection results when more than one field of a galaxy was observed, and also when there are several exposures within the same field. The comparisons allow us to estimate the internal accuracy of the ML method, the influence of signal-to-noise on the detection results, and the possible effects of population differences. For the comparison we selected the dIrr galaxy NGC 2366, for which there is an impressive quantity of HST/WFPC2 observational material in the HST archive. Two samples are appropriate for our purposes: a sequence of exposures with the F555W and F814W filters within program 8769 and pairs of exposures of a separate field in the F606W and F814W filters within program 9318. Details about exposures and the result of our measurements are given in Table 1.

The agreement of TRGB values in both fields is excellent; the difference is 0.02 mag. 
The first field covers the inner part of NGC 2366. The color-magnitude diagram of the sum of all images is presented in the top panel of Fig.10. The TRGB is situated well above the photometric limit in this case, and it is easily detected with our procedure. The color-magnitude diagram has huge blue and red supergiant populations, as well as rich RGB and AGB populations. The comparison of results between the summed photometry and the single exposure photometry in this inner field again demonstrates the internal accuracy is good: the mean deviation is 0.02 mag. The consequence of better signal with the summed data is negligible because the TRGB is already about 2 mag above the photometric limit with the individual exposures. The photometric results for the outer field of NGC 2366 are presented in the lower panel of Fig.10. There is only a small contamination here from red and blue supergiant stars, yet the AGB is well-populated in this outer region of the galaxy. The good agreement of the TRGB fits in the two fields demonstrates that the presence of a very prominent young population does not affect the TRGB measurement.

\subsection{Comparison of WFPC2 and ACS observations of the same galaxies}

With the new instrument Advanced Camera for Surveys (ACS) on HST, the capability to measure galaxy distances with the TRGB method has improved significantly. ACS provides a wider field of view, better resolution, and increased sensitivity. We can now obtain accurate galaxy distances at $10 \mathrm{Mpc}$ with one orbit of HST observing time. An example of the enhanced capability is provided by the dwarf galaxy KK 16 which was observed both with WFPC2 (program 8601) and ACS (program 10210). In the case of the WFPC2 observations (exp $=600 \mathrm{~s}$ in both $\mathrm{F} 606 \mathrm{~W}$ and $\mathrm{F} 814 \mathrm{~W}$ ), we find $\mathrm{F} 814 \mathrm{~W}_{T R G B}=$ $24.62 \pm 0.10(\mathrm{D}=5.0 \mathrm{Mpc}$ ) (lower panel Fig. 11). Definitely, this TRGB detection is near the limit for a WFPC2 snapshot observations. The ML procedure picks up the TRGB near the photometric limit where the photometric errors and bias are substantial. By contrast, 
the observations of the same galaxy with ACS (and slightly longer exposures of 1226s in $\mathrm{F} 814 \mathrm{~W}$ and $934 \mathrm{~s}$ in $\mathrm{F} 606 \mathrm{~W}$ ) give a very reliable CMD sampling with $\mathrm{F} 814 \mathrm{~W}_{T R G B}=$ 24.78 $\pm 0.04(\mathrm{D}=5.7 \mathrm{Mpc}$ ) (upper panel Fig. 11). The WFPC2 snapshot exposures only detect the onset of the RGB for KK 16, and the distance is underestimated. However, even in this extreme case the procedure does not fail egregiously.

There are circumstances that can give rise to wildly erroneous results. The problem is made clear in Figure 12 which provides a further comparison between WFPC2 and ACS observations. Measurements in good agreement lie along the 45 degree line. If the TRGB is bright then there is excellent agreement, but fainter than $I_{T R G B} \sim 24.5$ the WFPC2 values fall below the line.

With single-orbit WFPC2 observations, the photometric limit is $I \sim 25.5$. In the case of KK 16 that was just discussed, the TRGB is being identified but the location is biased by it's proximity to the photometric cutoff. On the other hand, in the cases of the four galaxies at the extreme right of the figure it is demonstrated by the ACS observations that the TRGB is below the WFPC2 detection limit. The WFPC2 measurements that have been reported for these galaxies (Karachentsev et al. 2003) are all based on the detection of the onset of the AGB.

We have not found an algorithmic way to ensure that the TRGB is being isolated and not the brightest AGB stars when the feature is near the photometric limit. The ratio of red stars to blue stars (cutting in color at $V-I=0.6$ ) in the first magnitude below the putative tip can give a good clue. AGB stars are usually (but not always) accompanied by substantial numbers of blue main sequence stars at comparable $I$ magnitudes. A sign of a dominant old population RGB appearing at the faint limit is a ratio of red to blue stars greater than 3.5. However, the only convincing way to establish if one is seeing the TRGB is to see the AGB above it. The onset of the AGB is $\sim 1$ mag brighter than the TRGB so 
should be evident if there is a chance to measure the TRGB.

The problems seen in the WFPC2 data will obviously also arise in the ACS data but, with single orbit observations, is displaced $\sim 1.5 \mathrm{mag}$ fainter. Problems will begin at $I_{T R G B} \sim 26$ due to the faintness limit at $\sim 27$. For the moment, there are hundreds of galaxies still to be observed with $I_{T R G B}<26$.

\subsection{Example of a difficult case}

The color-magnitude diagram of DDO 226 is demonstrated in Fig.13. Exposures of 600 sec were obtained in both F606W and F814W filters. This dwarf irregular galaxy has a substantial AGB population, which is clearly seen in the CMD, and a number of blue and red supergiant stars. The red giant branch and AGB are widened by errors because they are close to the photometric limit. The TRGB is situated about one magnitude above the

photometric limit. In this case the influence of photometric errors and incompleteness makes the TRGB detection especially difficult. However, using the photometric error modeling from the artificial star experiments still allows us to obtain reliable results: $\mathrm{F} 814 \mathrm{~W}_{T R G B}=$ $24.39 \pm 0.08$ at the $68 \%$ confidence level.

\subsection{Spatial selection in a difficult case}

When the TRGB is very close to the photometric limit, and the RGB is intrinsically contaminated by a significant population of intermediate-age or young stars, the detection of the tip can become very difficult or impossible. In these cases, knowledge of the spatial distribution of different stellar populations can be of great help in reducing the RGB contamination. As an example, Figure 14 shows the projected spatial distribution of all the detected objects around the galaxy UGC 3755, chosen from our ACS program 10210. 
The ellipse superimposed to the data has a major axis 0.014 degrees ( 50 arcsec), an ellipticity 0.9 , and a position angle 20 degrees. Left and right panels of Figure 15 show the corresponding CMDs for the regions inside and outside the ellipse, respectively. In this galaxy, as frequently occurs, young and intermediate-age stellar populations tend to be more centrally concentrated. As a consequence, the right panel of Figure 15 shows a much cleaner RGB, with very little contamination (note how the main sequence and supergiant sequences almost disappear). We could not obtain a satisfactory ML fit to the RGB tip with the CMD for the whole galaxy, but our ML procedure successfully measures the tip on the spatially filtered diagram, producing a value $I_{T R G B}=25.47 \pm 0.06(D=7.4 \mathrm{Mpc}$ with 0.17 reddening). This filtering procedure can successfully be applied to improve the precision of the TRGB determination as long as the number of stars in the first magnitude below the tip does not fall below $\sim 100$.

\section{Summary}

Let us briefly summarize the results of our study. The main aim of this work is the improvement of the TRGB estimation accuracy and reliability closer to the photometric limit. The necessity of a consistent methodology became evident during the analysis of our large stellar photometry database of HST/WFPC2 and ACS observations of nearby dwarf galaxies. The resulting procedure is a logical enhancement of previous work. The maximum likelihood algorithm is augmented with detailed modeling of photometric errors and the completeness function. The diverse testing of our method shows that it is reliable, has good accuracy, and does not introduce any systematic errors. This technique is especially useful in cases where the TRGB approaches the photometric limit and/or the RGB is poorly populated in the CMD.

The current study allowed the slope of the red giant branch luminosity function to 
be a free parameter in the maximum likelihood fits. However, in cases where the TRGB is within $\sim 1$ mag of the faint cutoff of the photometry the determination of the slope $a$ is unstable. Worse, there is a coupling between the slope and the location assigned to the TRGB. Because of the onset of incompletion, the ML fitting can spuriously chose a very flat or even negative slope for the LF and simultaneously prefer a value for the TRGB that is too faint. These points were confirmed with fitting to artificial CMD where the TRGB was precisely known. If the TRGB is well removed from the faint limit then it is possible to solve for the red giant LF slope with good accuracy. Inevitable, the slope is near to $a=0.3$ and the TRGB can be accurately measured assuming this value for the slope.

We further found that the slope $c$ describing the asymptotic giant branch luminosity function should be constrained. The slope of the AGB LF is less predictable. However, if left free, nonphysically large positive values for the AGB slope can mask the full amplitude of the TRGB jump. Experience tells us that the AGB slope lies in the range $0.2<c<0.4$.

Presently, we make ML fits using two alternative constraints on LF slopes. With the first alternative, the slopes for both the RGB and AGB are set at $a=c=0.30$. With the second alternative, the values of the slope parameters $a$ and $c$ are free but constrained by priors $a^{\prime} \pm \sigma_{a}=0.30 \pm 0.07$ and $c^{\prime} \pm \sigma_{c}=0.3 \pm 0.2$ where the primed values are the expectation slopes and $\sigma_{a}$ and $\sigma_{c}$ are the rms deviations around the expectation values found from 18 well observed LF. The preferred approach is the second alternative if there is a proper understanding of prior values and rms deviations and if the incompletion function is being properly formulated. We are considering the two alternative formulations in order to test for systematics once distance measures become available for the ensemble of galaxies suitably observed by HST.

The subject of the present study is just the detection of the TRGB in a colormagnitude diagram and the assignment of errors to the detection. The important error 
sources associated with calibration and extinction will be considered in Paper II. These potential sources of systematic error can contribute up to $5-10 \%$ uncertainty to the distance estimation (Bellazzini et al. 2001). Paper II will be concerned with the absolute value of the TRGB in $I$ and HST flight F814W passbands, the effects of metallicity in the absolute calibration, the effects of reddening, and the degree of agreement between the TRGB distance determinations and other methodologies.

We acknowledge use of the Hubble Space Telescope data archive at STScI, which is operated by the Association of Universities for Research in Astronomy, Inc. under NASA contract NAS 5-26555 and support from grants associated with HST programs AR-9950 and GO-9771, 10210, 10235. DM and LM are grateful to the Department of Astronomy, University of Maryland and Institute for Astronomy, University of Hawaii for hospitality during this work. This project also was supported by the Russian Foundation for Basic Research grant 04-02-16115. DIM acknowledge support from INTAS grant 03-55-1754 and from the Russian Science Support Foundation. DM is also grateful to Observatoire de Lyon for hospitality during my visits. 


\section{REFERENCES}

Baade, W. 1944, ApJ, 100, 137

Bellazzini, M., Ferraro, F., Pancino, E. 2001, ApJ, 556, 635

Bertelli, G., Mateo, M., Chiosi, C., \& Bressan, A. 1992, ApJ, 388, 400

Dolphin, A. E. 2000, PASP, 112, 1383

Dolphin, A.E. 2002, MNRAS, 332, 91

Dolphin, A.E. 2006, in preparation

Gallart, C., Aparicio, A., Vilchez J.M., 1996, AJ, 112, 1928

Girardi, L., Bressan, A., Bertelli, G., \& Chiosi, C. 2000, A\&AS, 141, 371

Holtzman, J., Afonso, C., \& Dolphin, A., 2005, in press

Iben, I., Renzini, A. 1983, ARA\&A, 21, 271

Karachentseva V.E., Karachentsev I.D. 1998, A\&AS, 127, 409

Karachentseva, V.E., Karachentsev, I.D. 2000, A\&AS, 146, 359

Karachentsev, I.D. 1994, Astron. Astrophys. Trans., 6, 1

Karachentsev, I.D., Dolphin, A., Sharina, M., Makarova, L., Makarov, D., Tully, R.B., Karachentseva, V., Sakai, S., Shaya, E.J. 2005, AJ, submitted

Karachentsev, I.D., Karachentseva, V.E., Huchtmeier, W.K., Makarov, D.I. 2004, AJ, 127, 2031 
Karachentsev, I.D., Sharina, M.E, Dolphin, A.E., Grebel, E.K., Geisler, D., Guhathakurta, P., Hodge, P.W., Karachentseva, V.E., Sarajedini, A., and Seitzer, P., 2003, A\&A, 398,467

Lee, M.G., Freedman, W.L., Madore, B.F. 1993, ApJ, 417, 553

Madore, B. F., \& Freedman, W. L. 1995, AJ, 109, 1645

Madore, B.F., Freedman, W.L. 1998, in Stellar astrophysics for the local group: VIII Canary Islands Winter School of Astrophysics, 263

Maíz-Apellániz, J., Cieza, L., MacKenty, J.W. 2002, AJ, 123, 1307

Meyer, M.J., Zwaan, M.A., Webster, R.L., et al. 2004, MNRAS, 350, 1195

Méndez B., Davis M., Moustakas J., Newman J., Madore B.F., Freedman W.L. 2002, AJ, 124,213

Rizzi, L., Held, E. V., Bertelli, G., Nasi, E., Saviane, I., \& Vallenari, A. 2002, ASP Conf. Ser. 274: Observed HR Diagrams and Stellar Evolution, 274, 490

Sakai, S. 1999, in IAU Symp. 183, Cosmological Parameters and the Evolution of the Universe, ed. K. Saito (Dordrecht: Kluwer), 48

Sakai, S., Madore, B.F., Freedman, W.L. 1996, ApJ, 461, 713

Sakai, S., Ferrarese, L., Kennicutt, R.C., Jr., Saha, A. 2004, ApJ, 608, 42

Salaris, M., Cassisi, S. 1997, MNRAS, 289, 406

Sandage A.R. 1971 in Proceedings of a Study Week on Nuclei of Galaxies ed. D.J.K. O’Connell (Amsterdam: North Holland, and New York: American Elsevier), p.601

Whiting, A.B., Hau, G.K.T., Irwin, M. 2002, ApJS, 141, 123 
This manuscript was prepared with the AAS LATEX macros v5.2.

Table 1: The TRGB detection results for NGC 2366

\begin{tabular}{lcccccc}
\hline Field & Filters & Exp. (sec) & $I_{\text {TRGB }}$ & $a$ & $b$ & $c$ \\
\hline Inner (sum) & F555W, F814W & 6700,4100 & $23.55_{-0.03}^{+0.04}$ & $0.40_{-0.02}^{+0.01}$ & $0.25_{-0.03}^{+0.03}$ & $0.54{ }_{-0.07}^{+0.07}$ \\
Inner (a) & F555W, F814W & 1300,1300 & $23.57_{-0.04}^{+0.03}$ & $0.38_{-0.02}^{+0.02}$ & $0.28_{-0.03}^{+0.04}$ & $0.48_{-0.07}^{+0.07}$ \\
Inner (b) & F555W, F814W & 1400,1400 & $23.56_{-0.04}^{+0.04}$ & $0.38_{-0.02}^{+0.02}$ & $0.25_{-0.03}^{+0.03}$ & $0.55_{-0.07}^{+0.07}$ \\
Inner (c) & F555W, F814W & 1400,1400 & $23.62_{-0.03}^{+0.04}$ & $0.35_{-0.02}^{+0.02}$ & $0.23{ }_{-0.03}^{+0.03}$ & $0.655_{-0.06}^{+0.07}$ \\
Outer & F606W, F814W & 1400,2000 & $23.56_{-0.06}^{+0.06}$ & $0.50_{-0.04}^{+0.05}$ & $0.39_{-0.09}^{+0.09}$ & $0.47_{-0.20}^{+0.22}$ \\
\hline
\end{tabular}



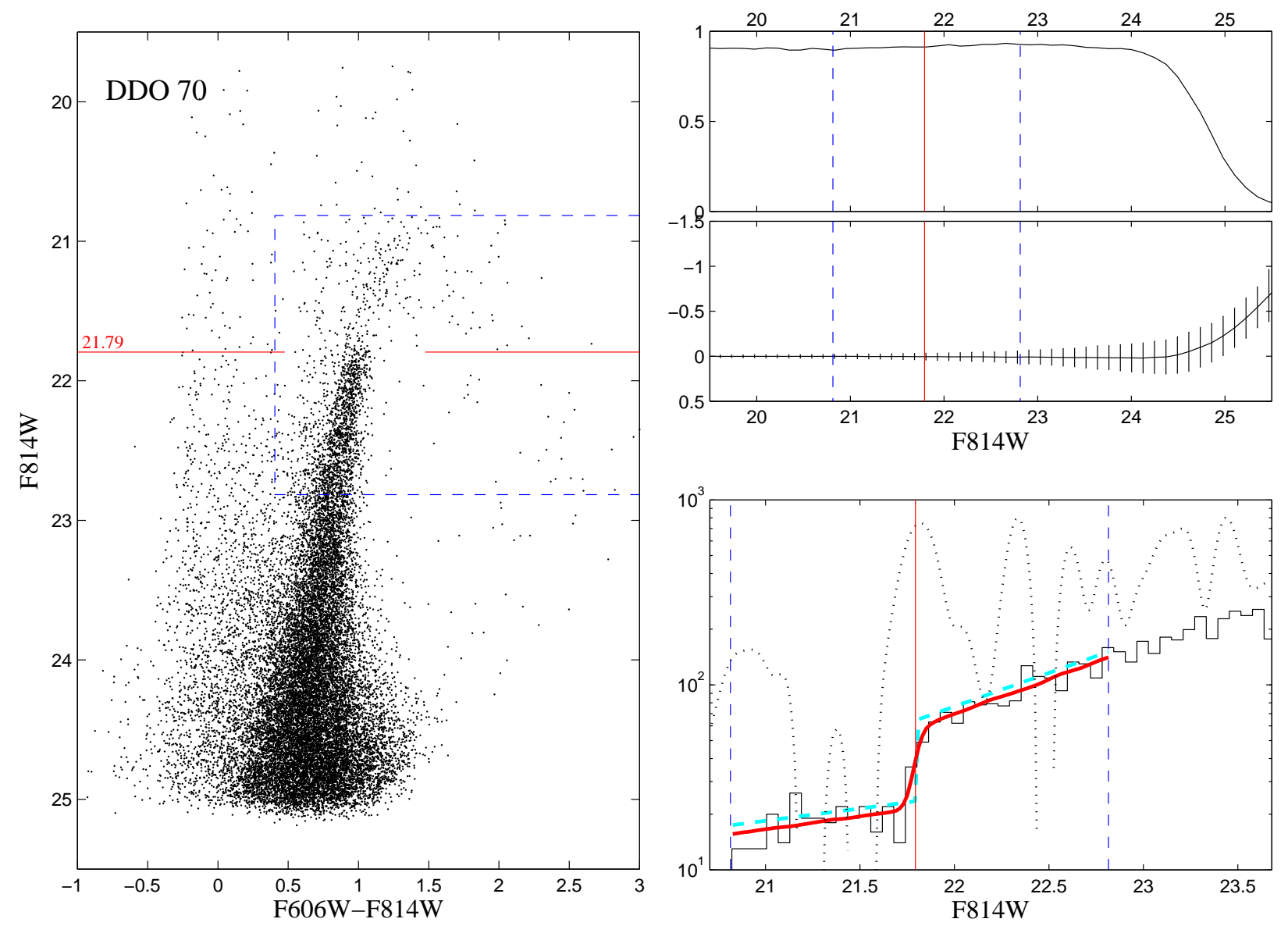

Fig. 1. - The color-magnitude diagram (left panel) and the TRGB calculation results for DDO 70. The upper right panel shows the completeness, photometric errors and dispersion in errors (vertical bars) vs. the HST WFPC2 F814W filter magnitude. The lower right panel gives a histogram of the F814W luminosity function. The Gaussian smoothed LF first derivative is shown as a faint dotted line. The resulting model LF convolved with photometric errors and incompleteness is displayed as a bold solid line. The intrinsic luminosity function associated with the model is plotted as the dashed line with a jump at the position of the TRGB. The fitted parameters are: $\mathrm{m}_{T R G B}=21.79[21.76,21.83]$, RGB slope $\mathrm{a}=0.36[0.30$, 0.43], RGB jump $b=0.44[0.38,0.50]$ and AGB slope $\mathrm{c}=0.13[0.03,0.24]$. 


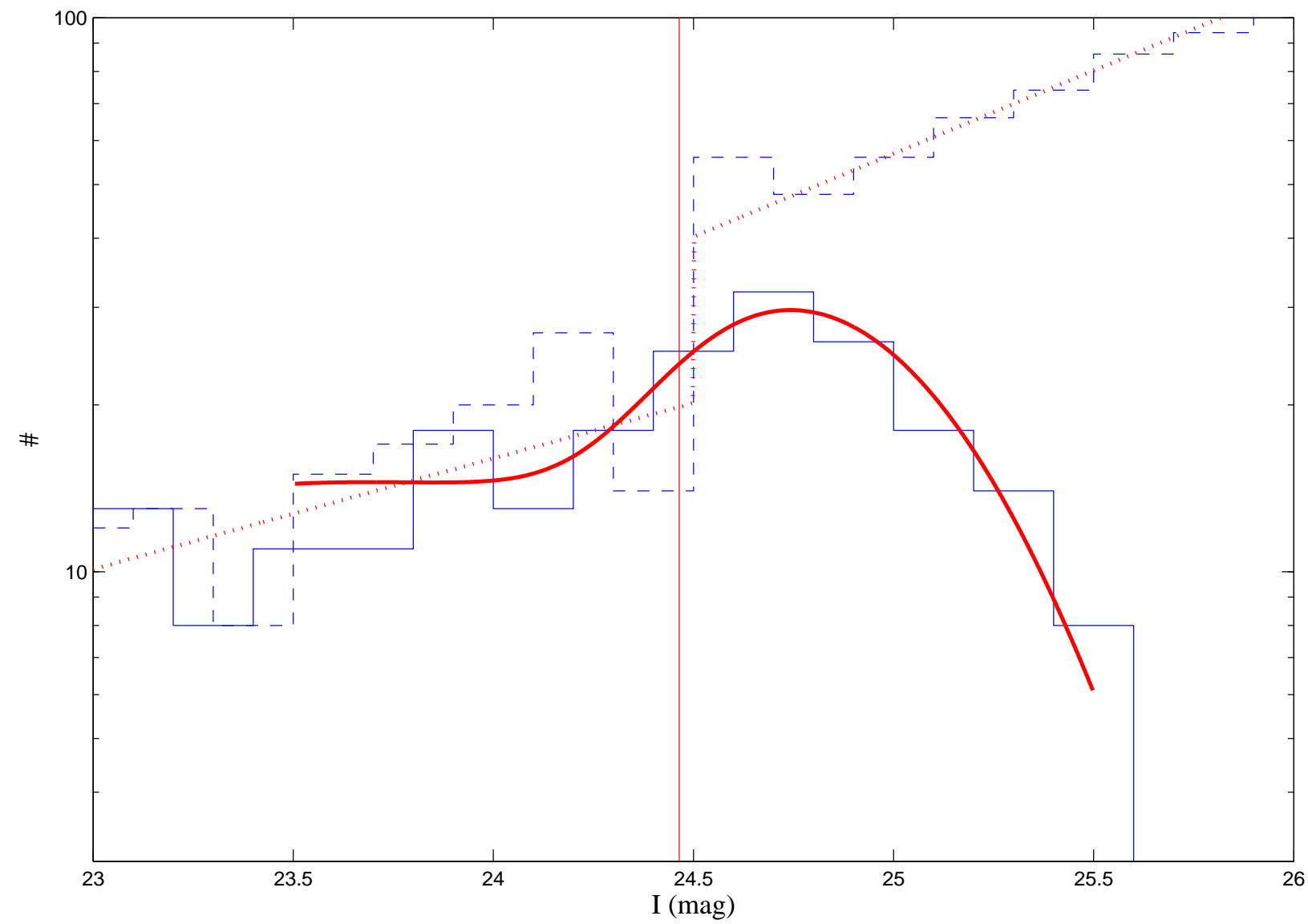

Fig. 2.- The luminosity function of an artificial star simulation. The input model LF is shown with the dotted line, the dashed histogram is a randomly generated realization of the input LF, the solid histogram is the LF after taking into account the observational errors, and the bold solid line represents the result of the maximum likelihood analysis. The vertical line indicates the recovered TRGB position. 


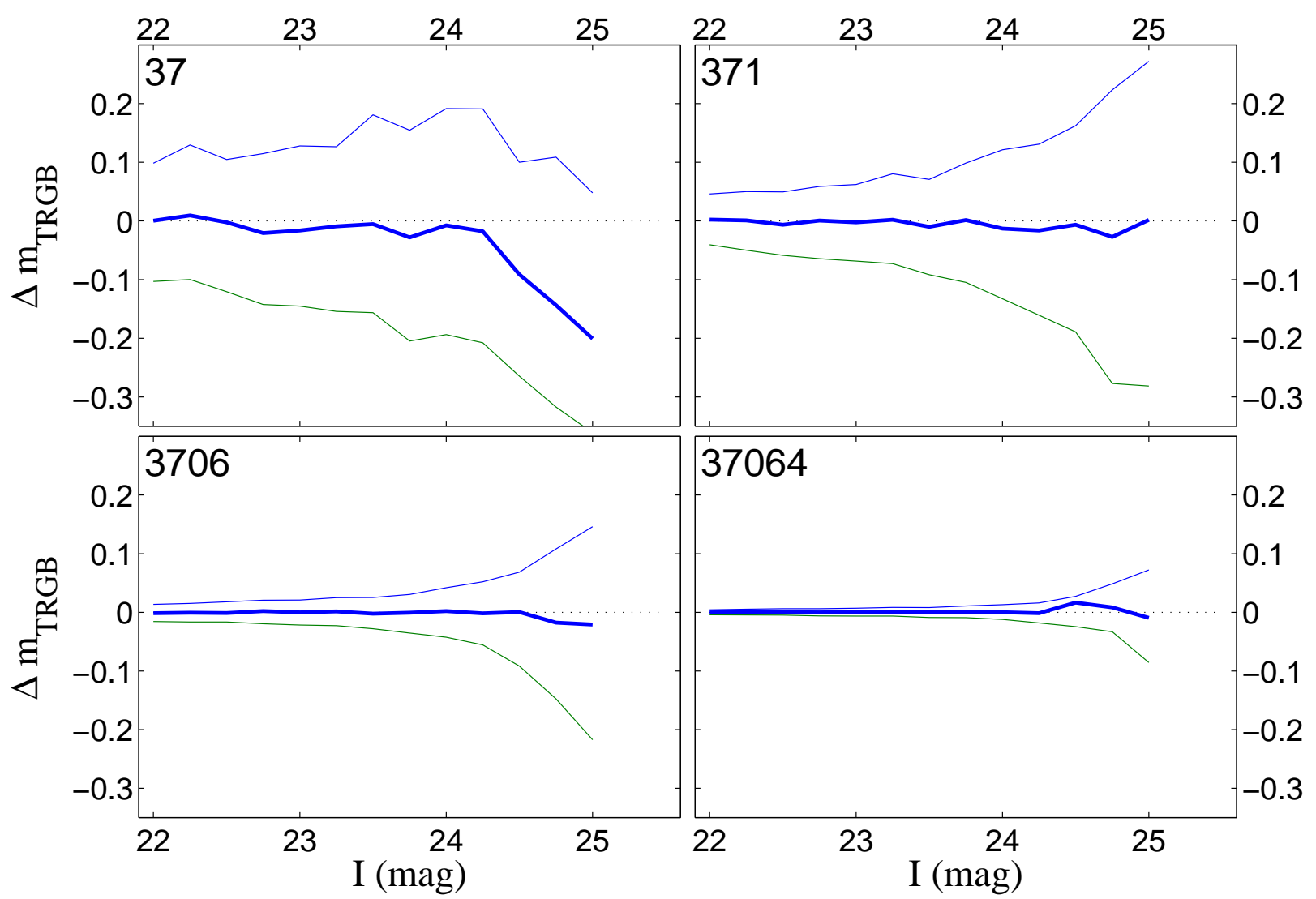

Fig. 3.- Median deviations of the recovered TRGB values minus input values are shown as bold lines. There are 37, 371, 3706, and 37064 stars within 1 magnitude of the TRGB in the four respective panels. The thin lines show 75th percentile high and low deviations. Experimental conditions reproduce snapshot WFPC2 observations. 

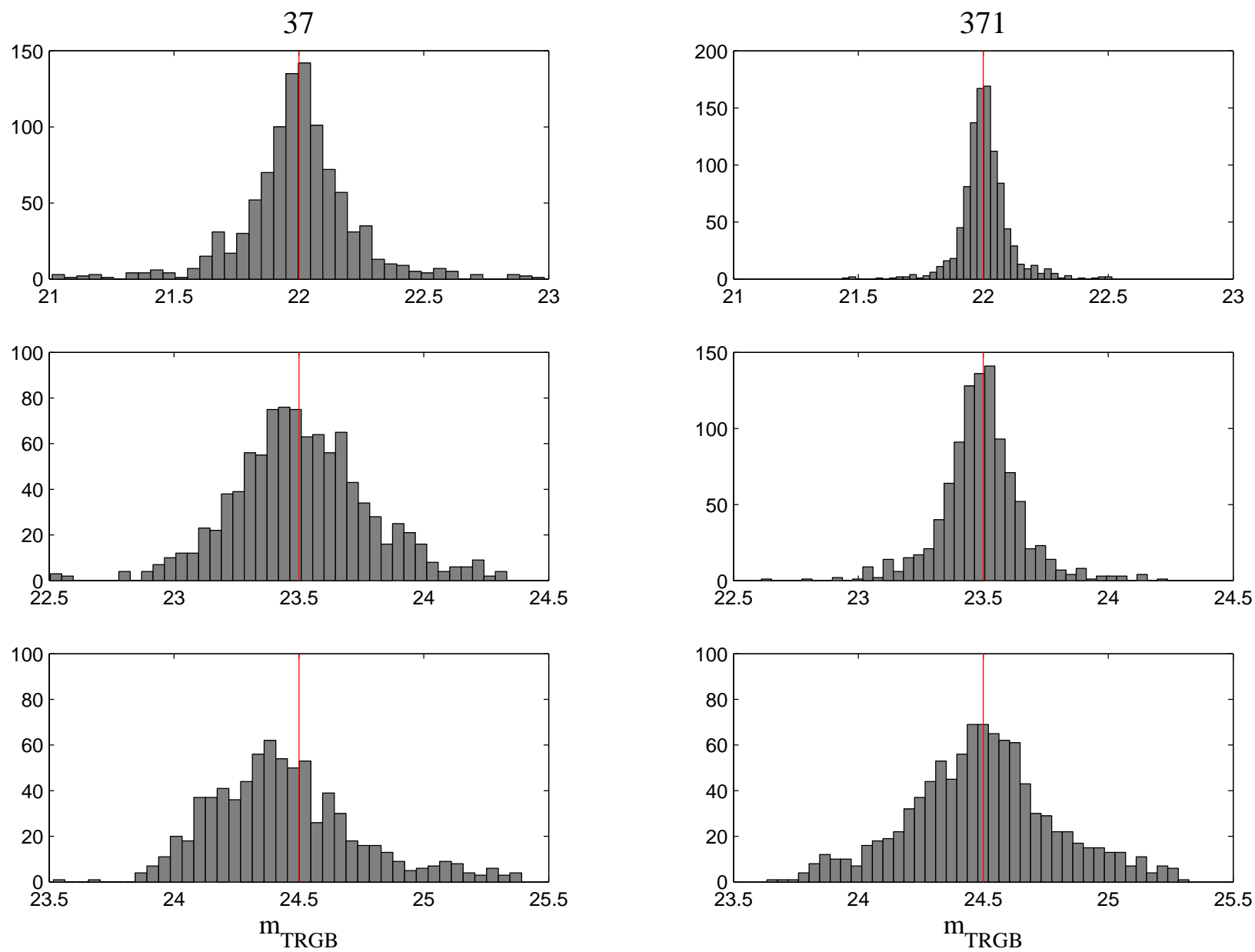

Fig. 4.- Distribution of reconstructed $I_{T R G B}$ for different sets of tests simulating snapshot WFPC2 conditions. The left panel shows the results for a sample with 37 stars within 1 mag of the TRGB and input $I_{T R G B}=22,23.5$ and 24.5. The right panel shows the results for a sample with 371 stars within 1 mag of the TRGB with the same input $I_{T R G B}$. 


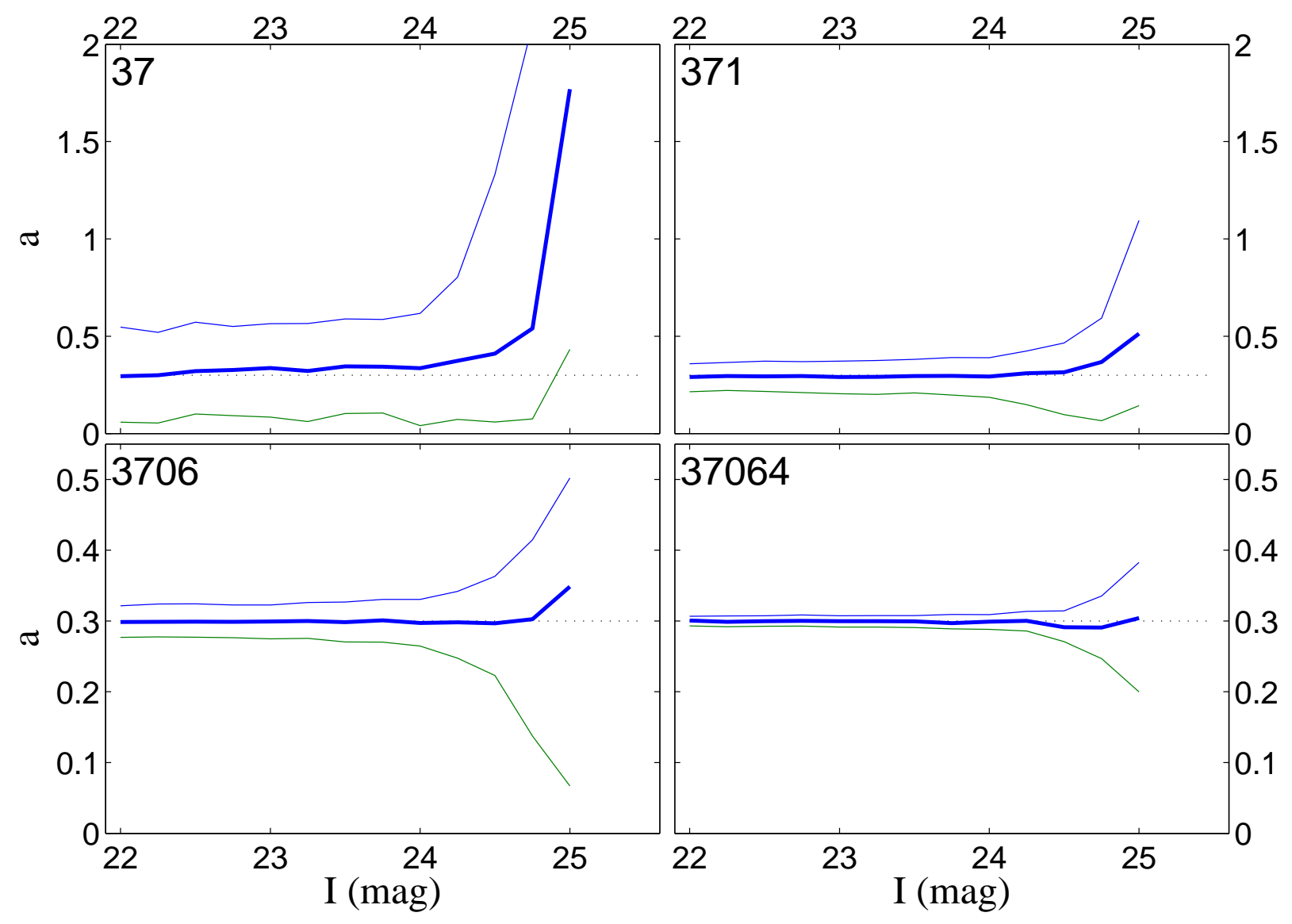

Fig. 5.- Recovery of the RGB slope parameter $a$ with snapshot WFPC2 conditions. The bold solid line is the median recovered value compared with the input value of 0.3 . The thin lines show 75th percentiles of high and low deviations. 


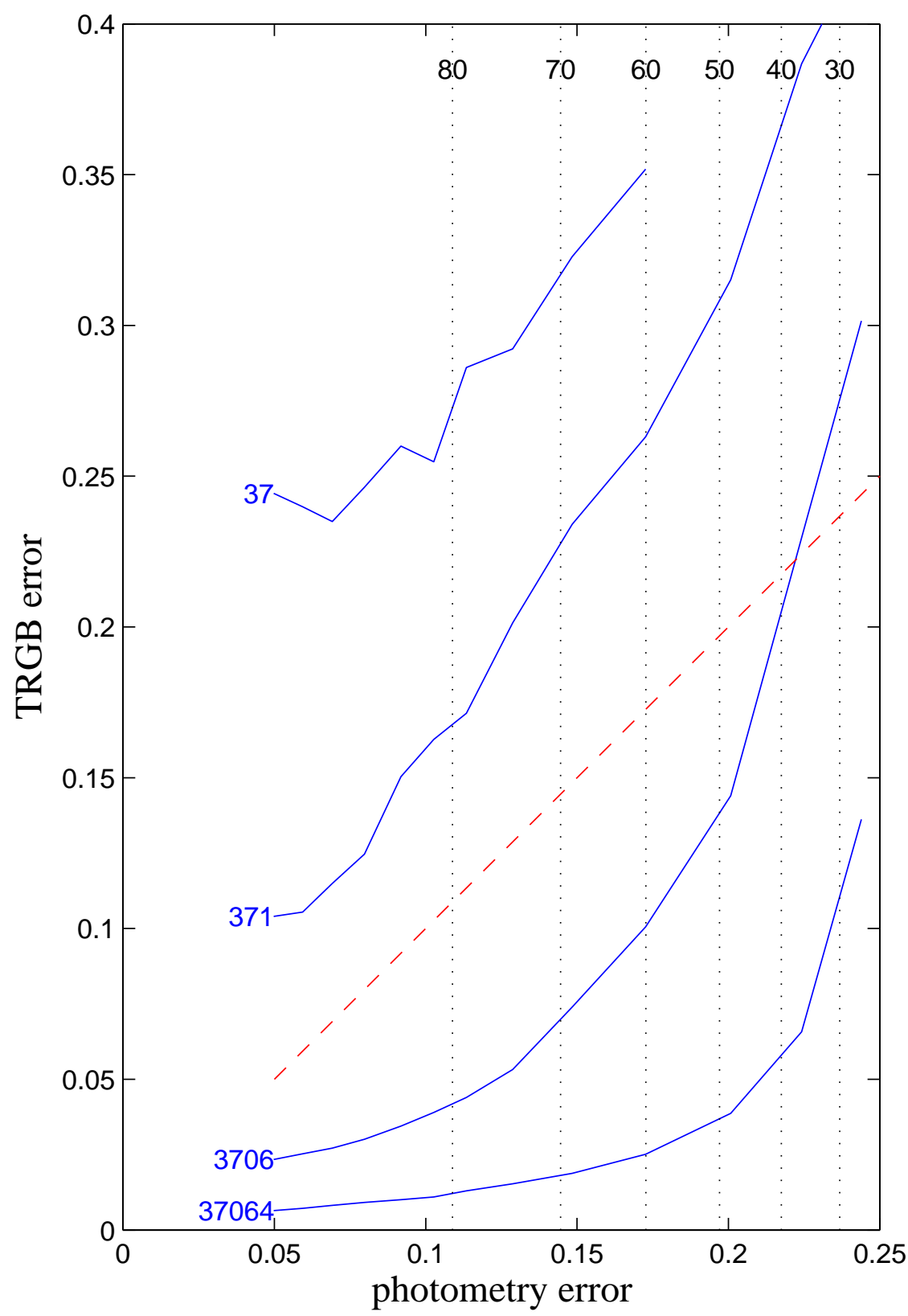

Fig. 6. - TRGB uncertainties vs. photometric error, received from artificial star experiments. The solid lines are mean TRGB uncertainties measured from Monte-Carlo simulations. Each line is marked with the mean number of artificial stars within 1 mag of the TRGB. The diagonal line is the line of equal errors of TRGB measurement and stellar photometric errors. The vertical dotted lines indicate the percent of stars still participating in the analysis after taking observational effects into account. 

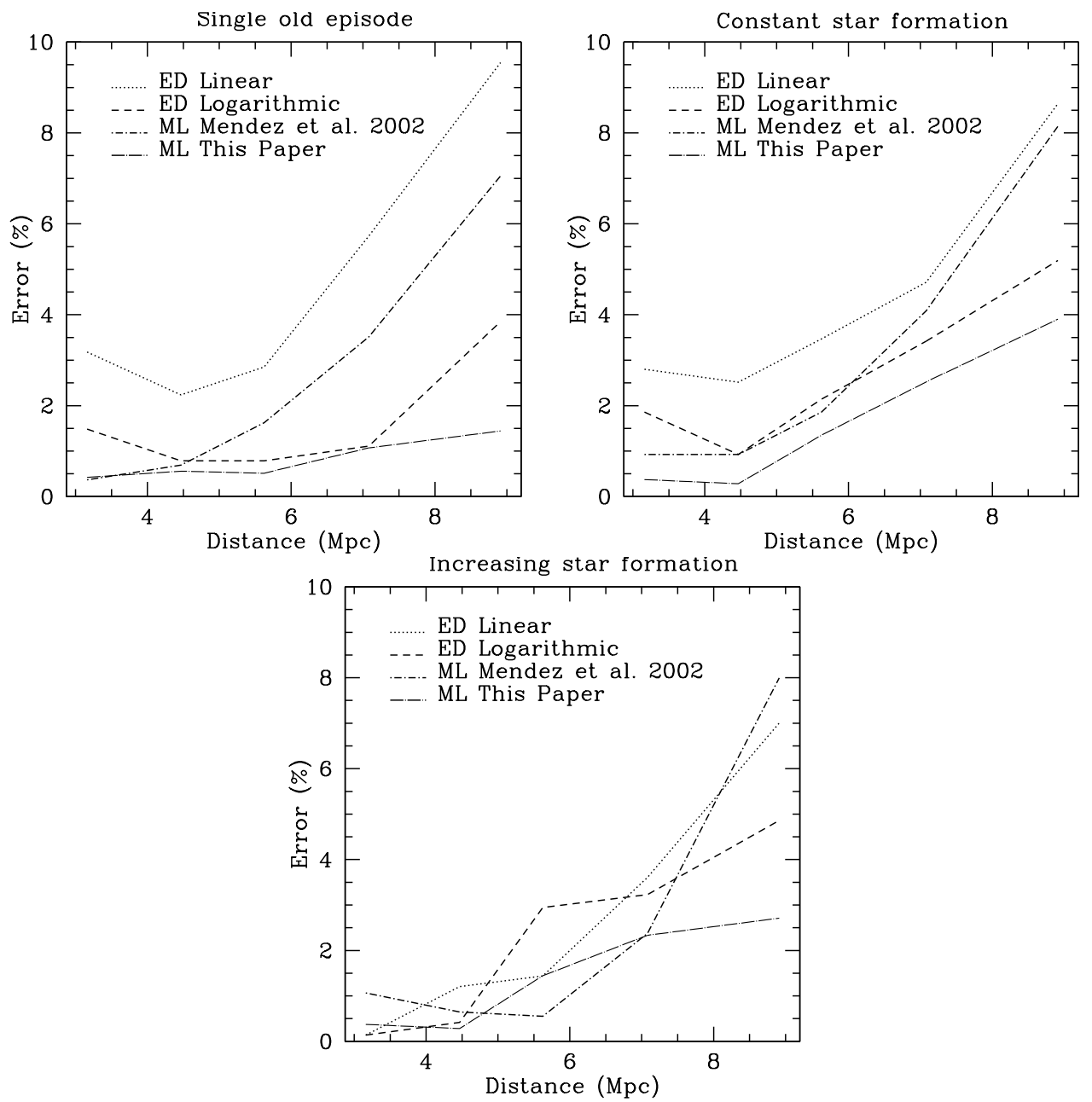

Fig. 7.- Contribution to the total distance error due to the TRGB detection alone. Top left panel: galaxies with a single old episode of star formation. Top right panel: galaxies with continuous star formation. Bottom panel: galaxies with an increasing star formation rate. The tests assume the sensitivity of single-orbit observations with ACS. 

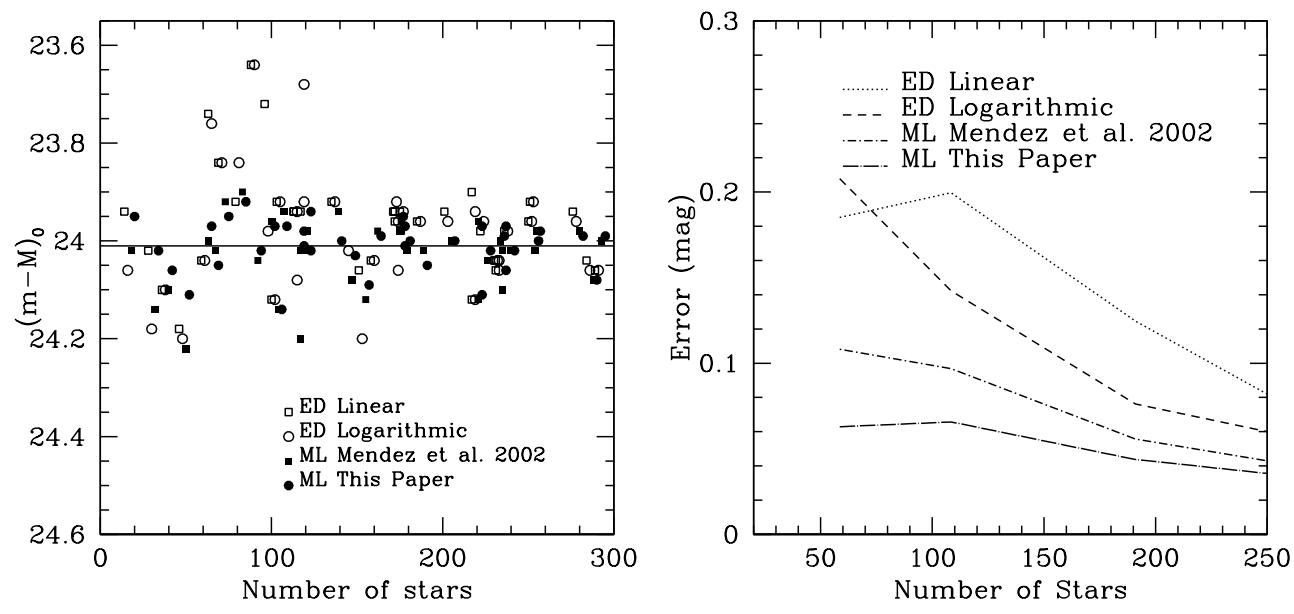

Fig. 8. - TRGB detection against the number of stars in the first magnitude bin. Left panel: deviations from the expected TRGB position at 24.01. Right panel: r.m.s. of the different methods against number of stars in the first magnitude bin. 


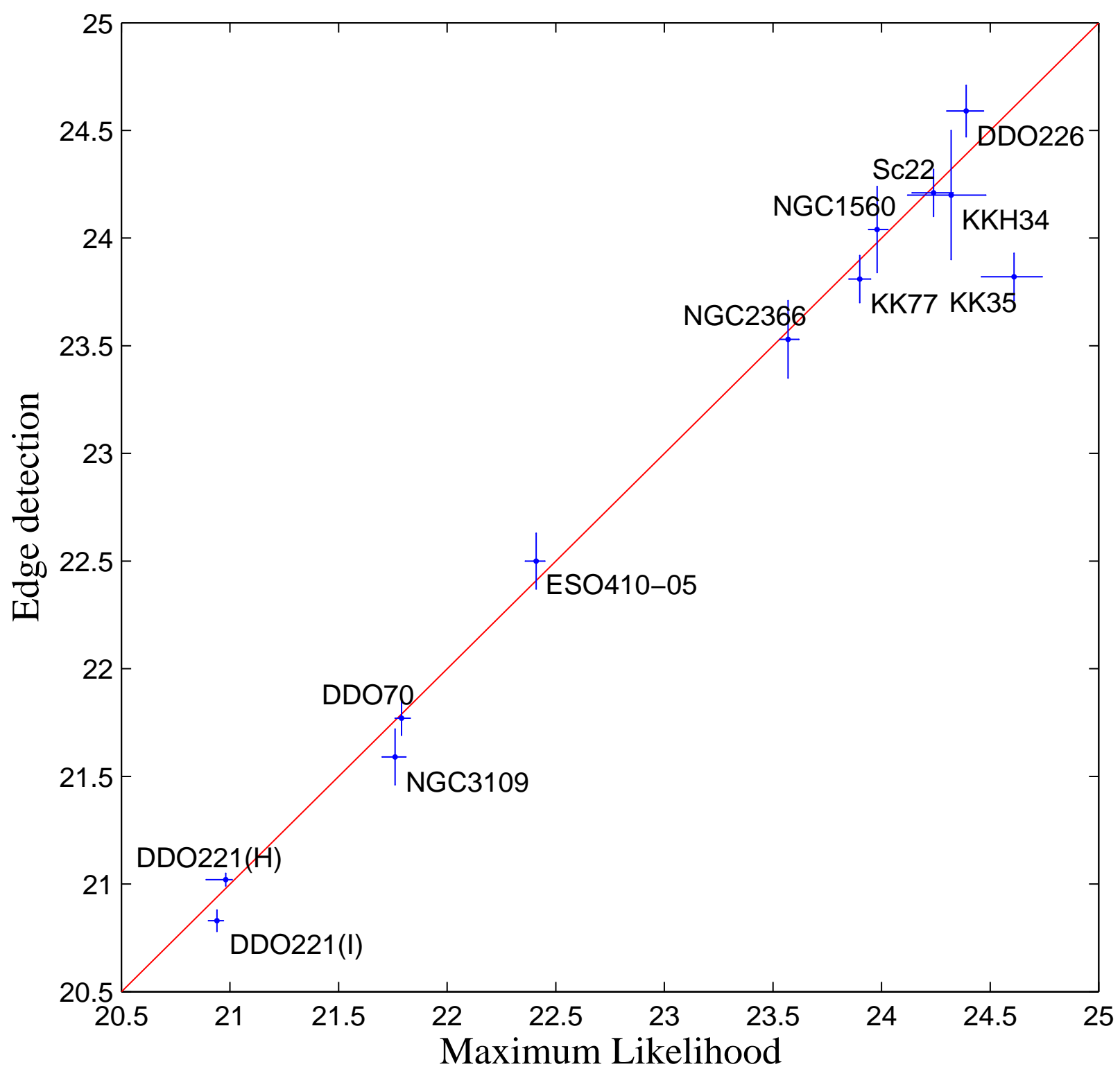

Fig. 9.- Comparison of TRGB detection results obtained alternatively with the maximum likelihood and edge detection algorithms. 

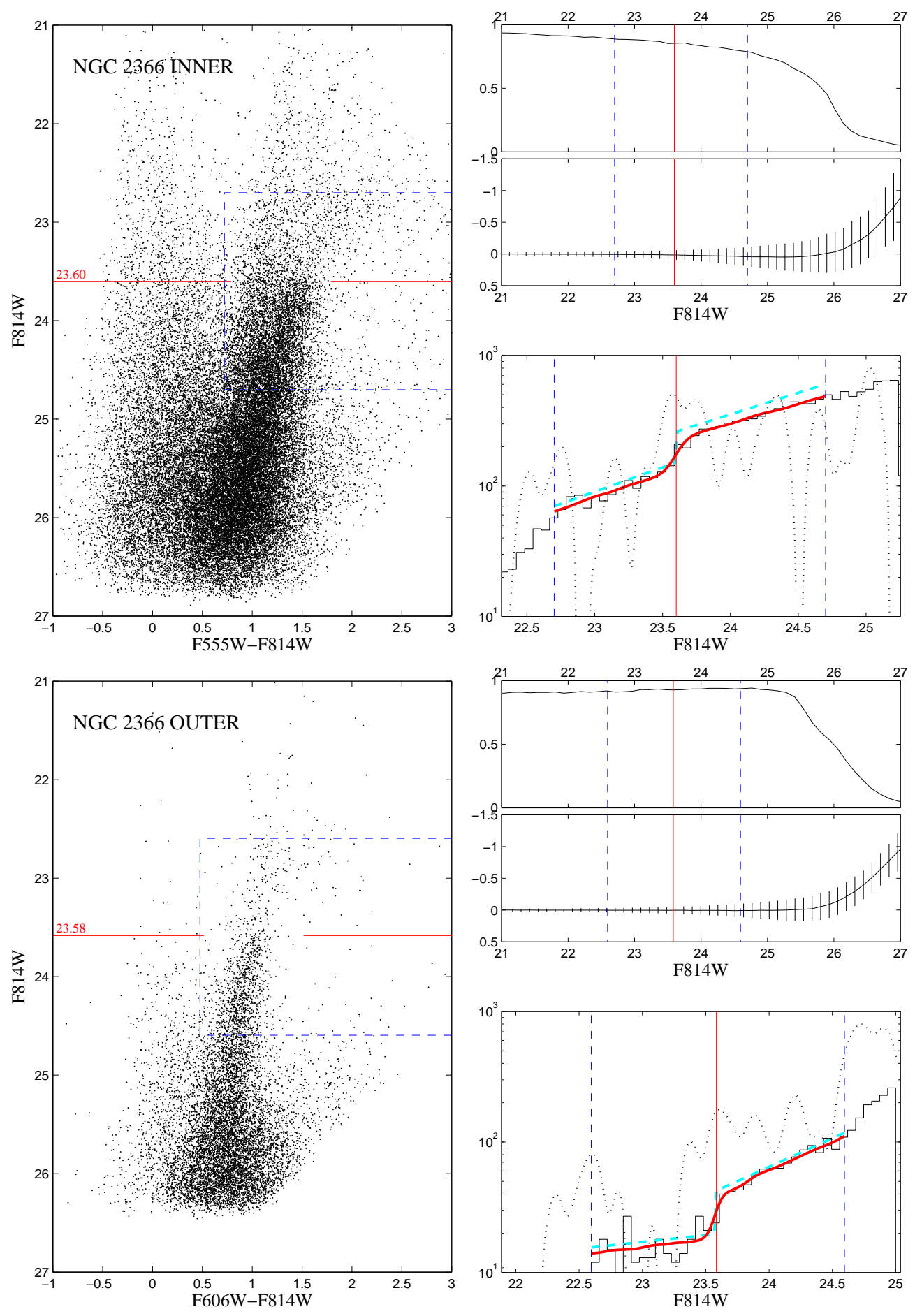

Fig. 10. - The TRGB detection result for the inner field of NGC 2366 (upper panel) and outer field of NGC 2366 (lower panel). The F814W vs. F555W-F814W or F606W-F814W (equivalent I,V-I) color-magnitude diagrams are at the left. Completeness, error, and error dispersion functions are at top-right. At lower-right, the stepped line is the histogram of the observed LF and the bold line is the LF accounting for uncertainties and incompletion. 

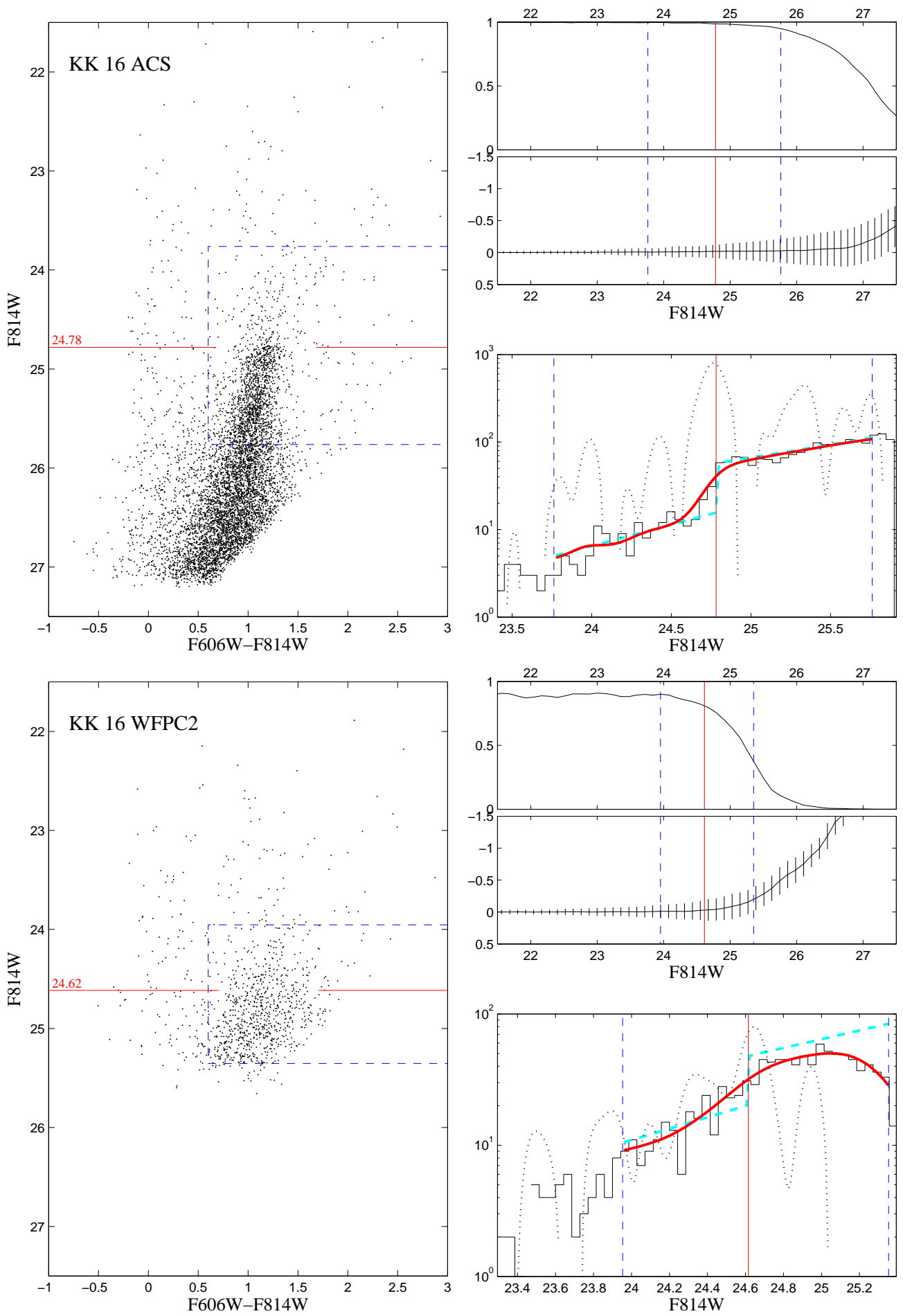

Fig. 11. - The color-magnitude diagram and the TRGB calculation results for KK 16 ACS observations (upper panel) and the same for KK 16 WFPC2 observations (lower panel). 


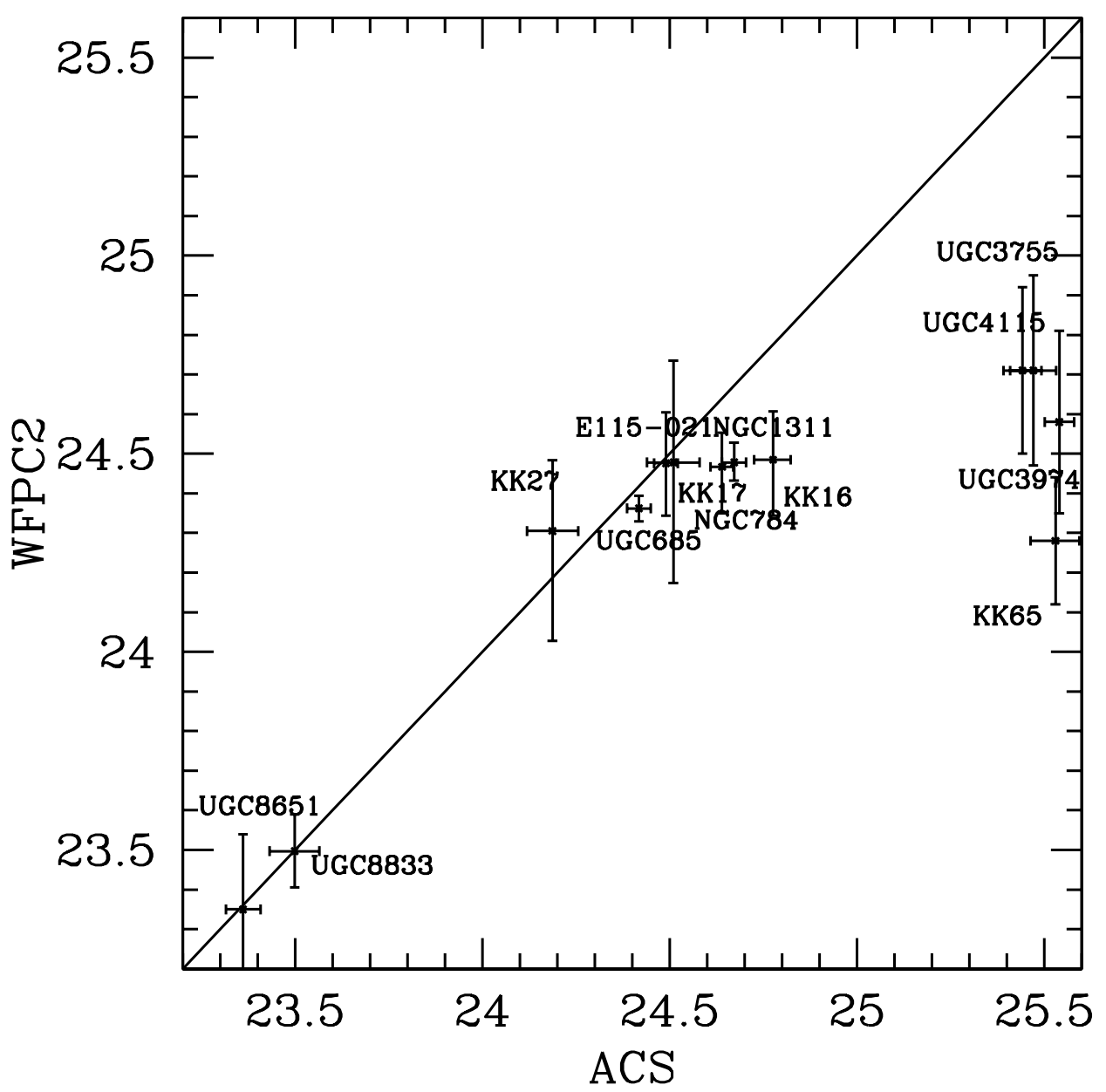

Fig. 12.-Comparison of single orbit ACS and WFPC2 TRGB determinations. Single orbit WFPC2 measures are unreliable at $I_{T R G B}>24.5$. It is expected that single orbit ACS measures become unreliable at $I_{T R G B}>26$. 

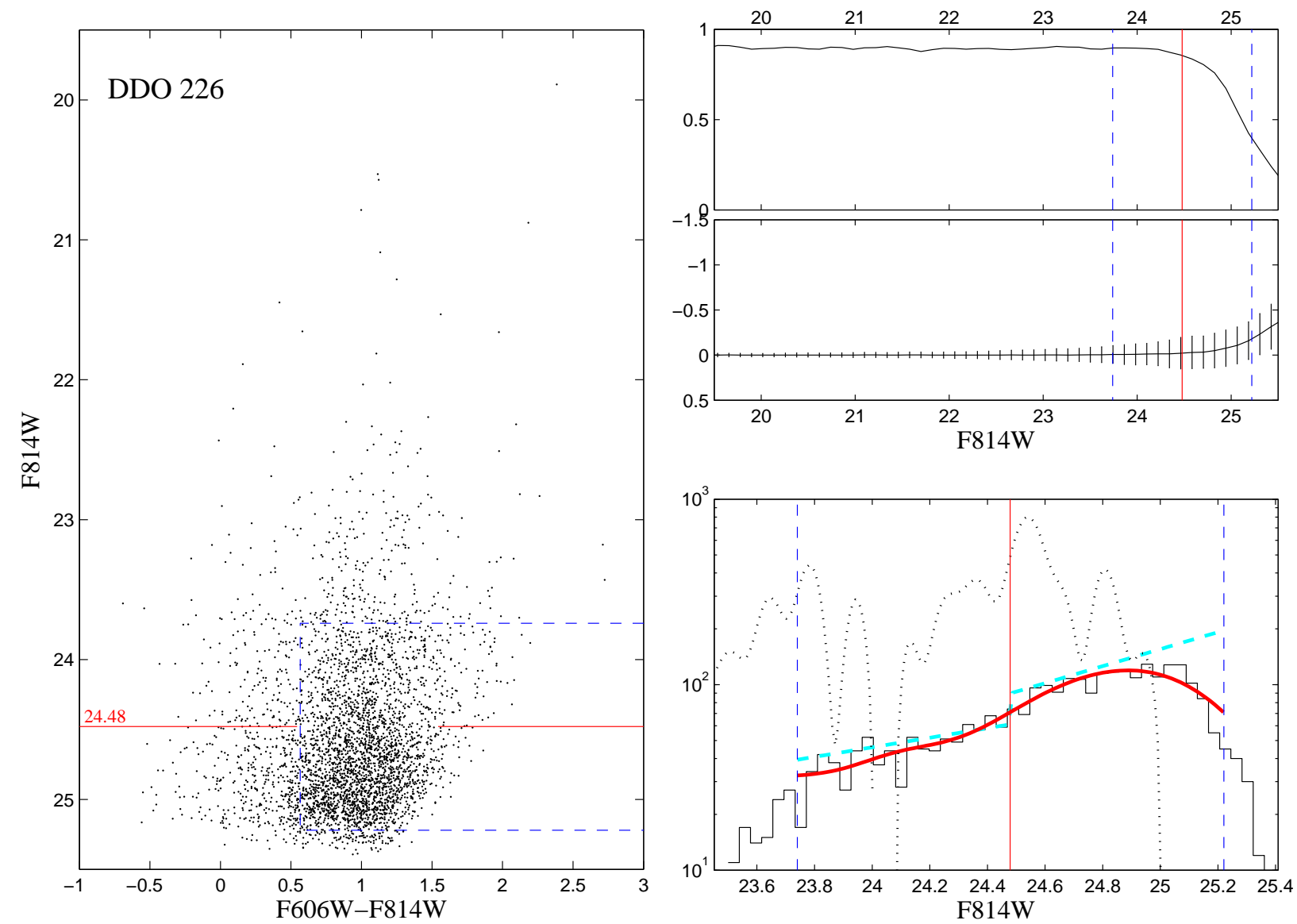

Fig. 13. - The color-magnitude diagram and the TRGB calculation results for DDO 226. The upper right panel demonstrates the completeness, photometric errors, and dispersion (vertical bars) vs. F814W mag. The lower right panel is a histogram of the F814W luminosity function (solid line), Gaussian smoothed LF first derivative (dotted line), the model LF accounting for errors and incompletion (bold solid line), and the corresponding intrinsic LF (dashed line with step at the TRGB). 


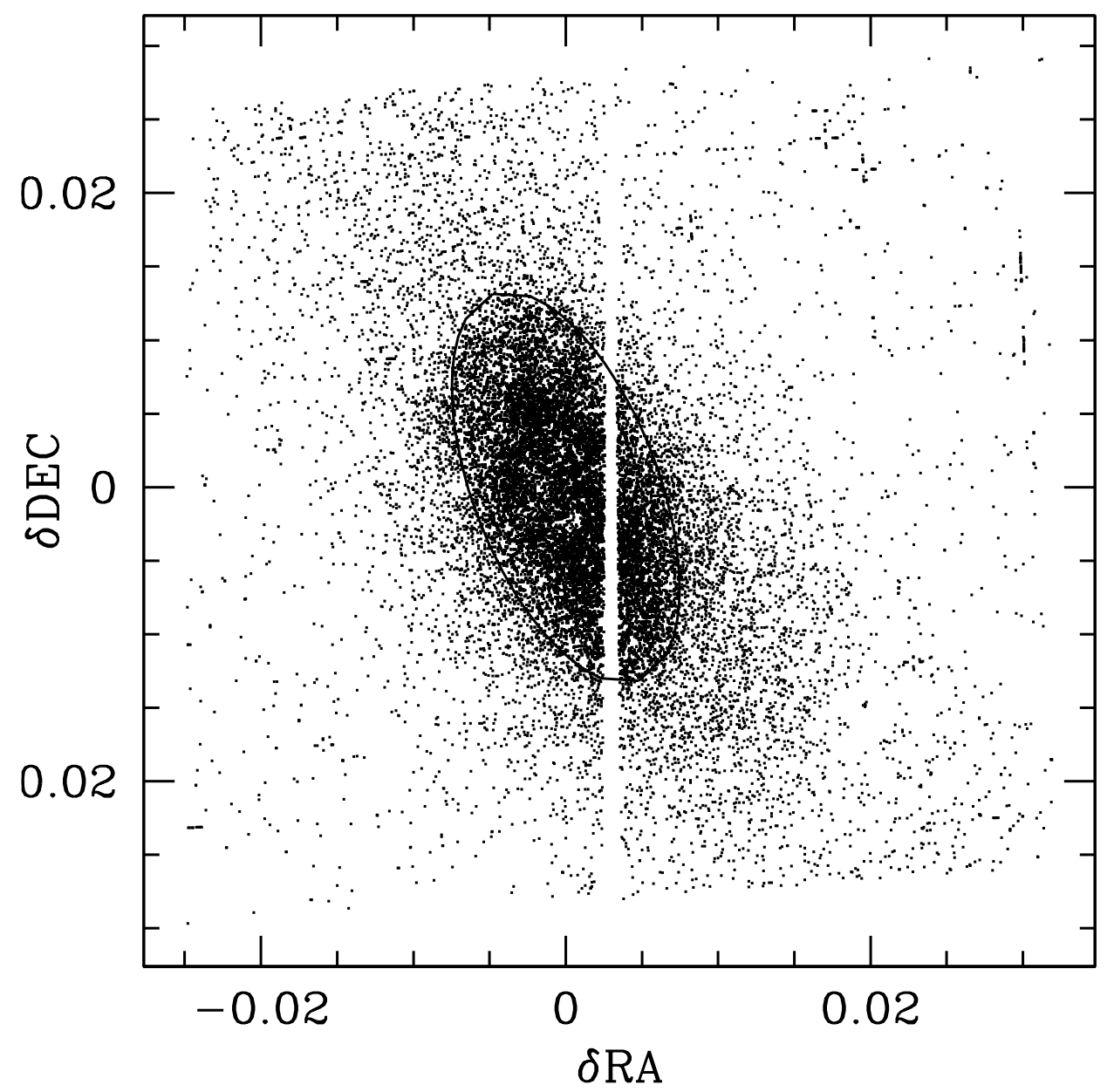

Fig. 14.- Projected spatial distribution of detected objects around UGC 3755 from ACS observations. 


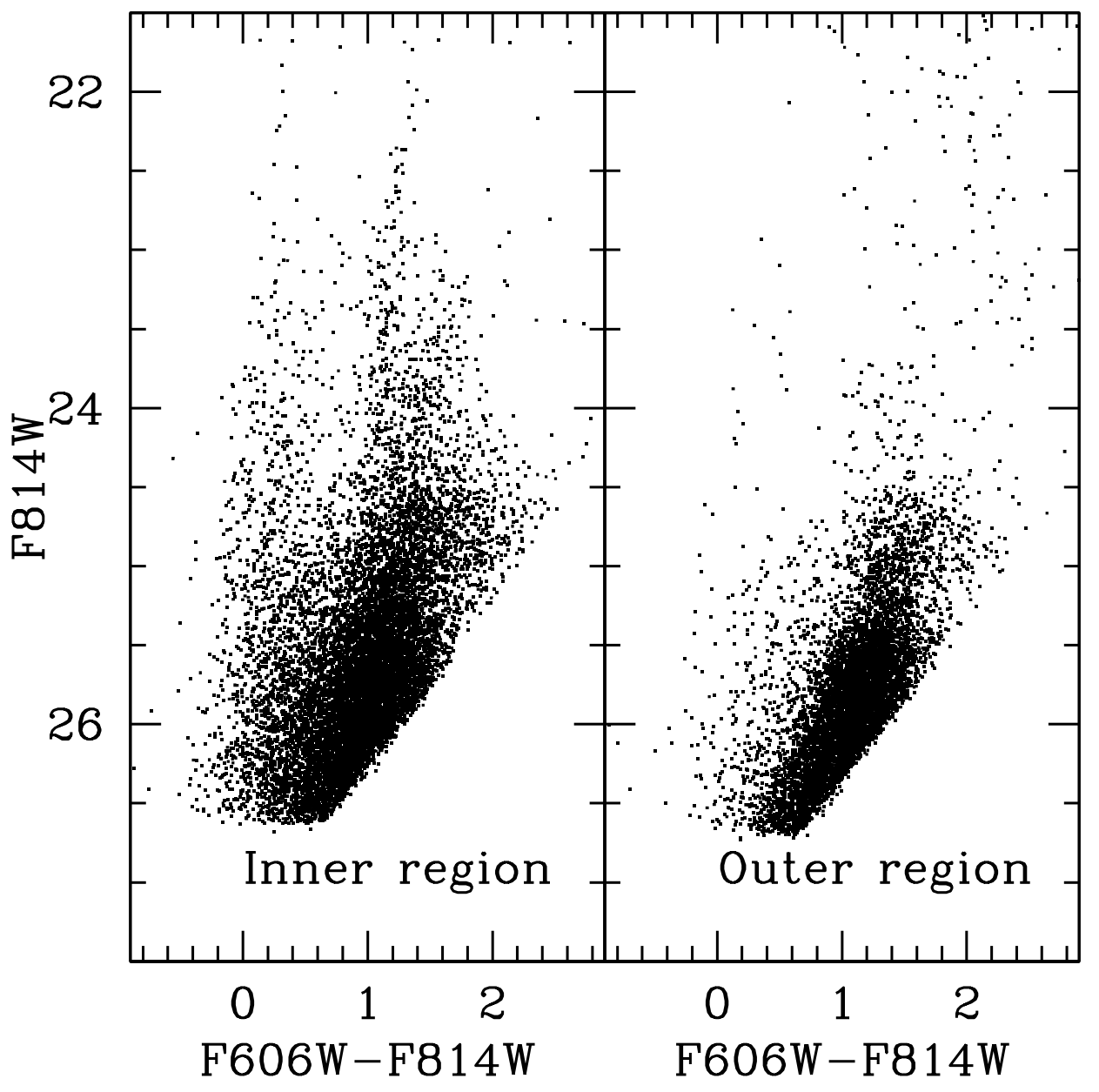

Fig. 15.- Left panel: CMD of UGC3755 in the region inside the ellipse of Figure 14. Right panel: CMD of UGC3755 in the region outside the ellipse. 